\title{
The Treatment Planning of Rectum Tumors and The Use of The Wedge Filter for Ensuring The Correct Isodoses
}

\author{
Idajet Selmani and Partizan Malkaj
}

\begin{abstract}
One of the most important issues in the field of radiotherapy is the correct distribution of the dose around the volume of interest or planning target volume (PTV). For making this possible the exact isodose in a treatment plan has to cover the PTV, so it is used the wedge which is a part of the linear accelerator head. Wedge plays the role of a filter and usually it is called wedge filter. The wedge filter is in use almost in all treatment plans, for all the parts of the body. In this paper it is consider the use of the wedge filter for treatment of rectum tumors.

The process starts with the scanning of the patient and the deliantion of the interest's volums in the Monaco system. In the following the imagins have been sent in the treatment planning system for making the nesessary plans for treatment of the rectum. Two plans were done, one with the use of the wedge and the other without using it. The dose volume histogram helps for compering the results of the plans. The best conformity of the isodoses it was for the plan with the use of wedge through volume of interest, which is planning target volume (PTV).
\end{abstract}

Keywords-Accelerator, rectum tumor, treatment planning, wedge.

\section{INTRODUCTION}

Radiotherapy aim is giving the correct dose to the PTV and protecting maximally the organs at risk and the healthy tissue around PTV. This is achieved by having the correct isodose line in the target. This homogeneity is not easy to be gain. Wedge filters were first conceived and used in radiotherapy by Frank Ellis, 1944. The intensity of the beam is reduced gradually by a filter in a form of a wedge across the radiation field to shape the isodose curves. The wedge was placed between the patient and the source head at a fixed distance [1].The work done for this paper it is focused in the plans for rectum tumors. Because the irradiating fields are one posterior and two laterals there is not uniformity of the isodoses in the PTV. Some parts of PTV are irradiated with more than the prescribed dose line and some with less. In these cases the wedge is a big help to create a more correct uniform isodose around PTV. For the same patients there were created two plans, one without wedge in the path of the beam and the other with wedge in path of the beam.

\section{METHODS AND MATERALS}

In radiotherapy wedge are placed for modifying the path of photon beams in a way to have the correct isodose distributions (Fig.1).

Published on February 22, 2020.

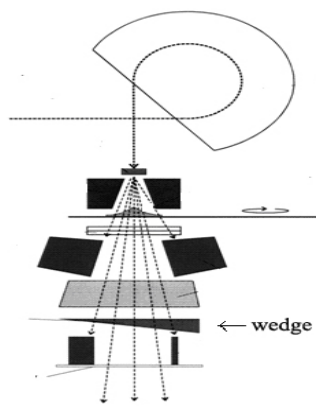

Fig. 1. The wedge in the path of the beam

The modified isodose distributions are invaluable in the treatment planning to achieve homogeneous isodose distributions. A wedge filter, which is usually made of dense materials such as steel, lead, brass, copper or any other heavy metal, is designed to create differential attenuation and hence progressive change in the intensity across the photon beam width. The resulting isodose distributions present tilting of isodose curves with respect to the normal of the photon beam central axis. The degree of isodose curve tilt towards the thin end of the wedge filter depends on the physical slope of the wedge filter.

Rectum cancer is a common tumor for both men and women. The deaths are reduced significantly due to radiotherapy [2]. Plan of patient with rectum tumor have been studied in this paper. The main point is to show effect of the use of the wedge filter in the dose distribution through rectum volume. Before in radiotherapy, by using the physical leaf or by reducing the size of the field, it was achieved the wedge effect during the course of treatment [3]. Now the physical wedge in the linear accelerators is as standard accessory. The images were sent to the Monaco after the patients have been scanned in the CT simulator [4]. The tumor and the organs at risk are contoured by the radiotherapeud in the Monaco system. The belly step is accessory which was used to immobilize the patient. In this kind of tumors the organs at risk which should be protected are bladder, intestine and femur heads [5]. After finishing with contouring starts the work for creation of the plans in the treatment planning system [6]. There were created two different plans. The first with tree beams, one posterior and two laterals beams. The normalization point is the same for the three beams. In this case it was not in use the wedge (Fig. 2). The isodose $110 \%$ cover a big part of the PTV which means $10 \%$ dose more than prescription. So some part of the PTV is covered with more than prescribed dose by the doctor and some with much less than the prescribed dose. This will lead to a non good result in the treatment of the tumor. 


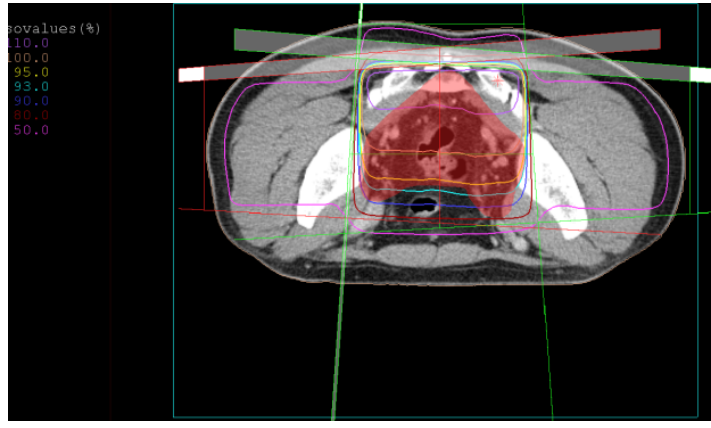

Fig.2. The plan for rectum without wedge filter

In the second method there are wedges in the path of the beam. The directions of the beams were exactly the same like the first method (Fig.3). The isodose $110 \%$ it is disappear in the volume of PTV. So we have $100 \%$ isodose around the PTV. This is the way for controlling maximally the rectum tumors, because all the part of the interest volume takes the correct isodose.

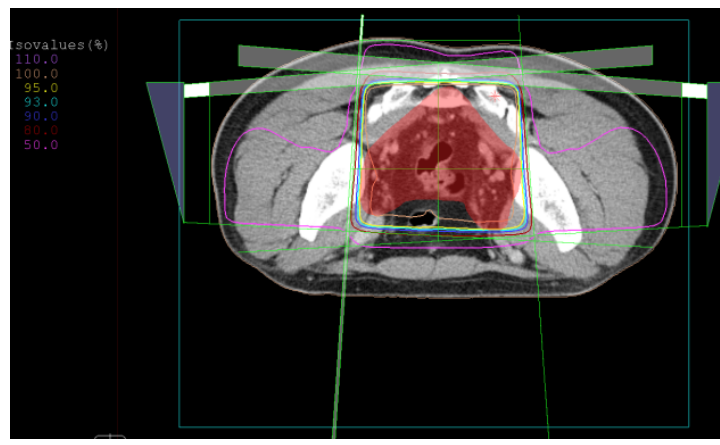

Fig. 3. The plan for rectum with wedge filter

\section{RESULTS AND DISCUSSION}

In the field of radiotherapy the wedge filter is the most commonly used beam modifier. In the treatment planning the use of wedge in radiation is numerous. One of the goals is to have uniform dose in the tumour volume in cases where the use of normal fields is hard to achieve for different reason.

The number of patients diagnosed with rectum tumor is getting higher recently. In mean time the radiotherapy tries to be more effective to resolve such problems with the help of treatments plans which are been created for every patients. The most important part of the procedure is to have uniform dose in all PTV. In this paper there were done two plans for the same patient, one with wedges in the path of the beam and the other without it. The plans were compared according to the dose volume histograms. From the observation it was noticed that the plan in which was not used the wedge (the solid line) has some part of PTV irradiated with more than prescribed dose and the other part with lower dose, which means the uniformity is bad. On the other hand the other line shows that the distribution in the PTV is uniform and under the correct dose which should be delivered to the patient (Fig. 4).

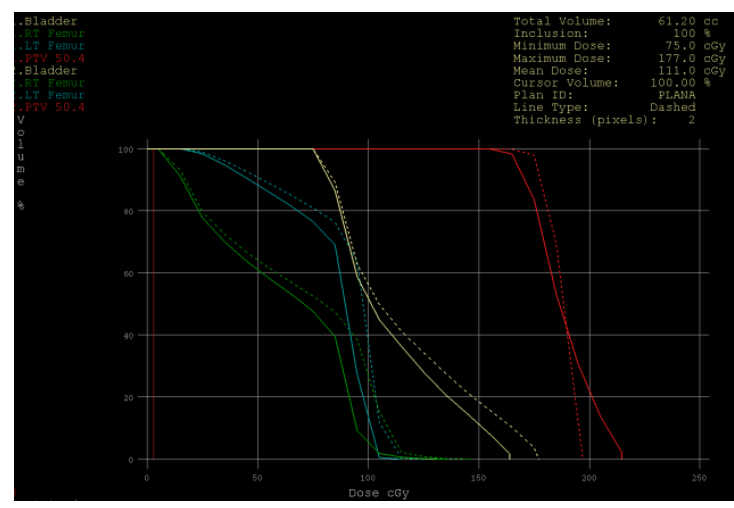

Fig.4. The comparison of histograms

This action is important and ensured that more precise to be in the giving of the correct isodose line around PTV more the control will rise to the tumor. The wedge helps for compensating for the irregular shape of the isodoses. The correct irradiation of the rectum volume brings the best control to the tumor.

\section{REFERENCES}

[1] Ellis F, and Miller H. The use of wedge filters in deep x-ray therapy. British Journal of Radiology 17:904 (1944).

[2] NIH consensus conference Adjuvant therapy for patients with colon and rectal cancer. JAMA. 1990;264:1444-50. [PubMed]

[3] Swedish Rectal Cancer Trial. Cedermark B, Dahlberg M, et al. Improved survival with preoperative radiotherapy in resectable rectal cancer. N Engl J Med. 1997;336:980-7. [PubMed]

[4] P. Cherry, A. Duxbury (1998). Practical radiotherapy physics and equipment, 139. Cambridge University Press.

[5] Stephens RJ, Thompson LC, Quirke P, et al. Impact of short-course preoperative radiotherapy for rectal cancer on patients' quality of life: data from the Medical Research.

[6] W. Parker, H. Patrocinio (2005). Clinical treatment planning in external photon beam radiotherapy. In: Radiation Oncology Physics: a handbook for teachers and students (E. B. Podgorsak. (Ed)), 219. International Atomic Energy Agency, Vienna. 